\title{
A Chinese Herbal Medicine, Jia-Wei-Xiao-Yao-San, Prevents Dimethylnitrosamine-Induced Hepatic Fibrosis in Rats
}

\author{
Shu-Chen Chien, ${ }^{1,2}$ Wei-Chiao Chang, ${ }^{1,3,4,5,6}$ Pu-Hua Lin, ${ }^{7}$ Wei-Pin Chang, \\ Shih-Chung Hsu, ${ }^{9}$ Jung-Chen Chang, ${ }^{10}$ Ya-Chieh Wu, ${ }^{11}$ Jin-Kuo Pei, ${ }^{12}$ and Chia-Hsien Lin ${ }^{12}$ \\ ${ }^{1}$ Department of Clinical Pharmacy, School of Pharmacy, Taipei Medical University, No. 250, Wuxing Street, \\ Xinyi District, Taipei 110, Taiwan \\ ${ }^{2}$ Department of Pharmacy, Taipei Medical University Hospital, No. 252, Wuxing Street, Xinyi District, Taipei 110, Taiwan \\ ${ }^{3}$ Department of Pharmacy, Taipei Medical University-Wan Fang Hospital, No. 111, Section 3, Xinglong Road, \\ Wenshan District, Taipei 116, Taiwan \\ ${ }^{4}$ Master Program for Clinical Pharmacogenomics and Pharmacoproteomics, School of Pharmacy, Taipei Medical University, \\ No. 250, Wuxing Street, Xinyi District, Taipei 110, Taiwan \\ ${ }^{5}$ Graduate Institute of Clinical Medicine, College of Medicine, Kaohsiung Medical University, No. 100, \\ Shiquan 1st Road, Sanmin District, Kaohsiung 807, Taiwan \\ ${ }^{6}$ Cancer Center, Kaohsiung Medical University Hospital, No. 100, Tzyou 1st Road, Kaohsiung 807, Taiwan \\ ${ }^{7}$ Food and Drug Administration, Ministry of Health and Welfare, No. 161-2, Kunyang Street, Nangang District, \\ Taipei 115, Taiwan \\ ${ }^{8}$ Department of Healthcare Management, Yuanpei University, No. 306, Yuanpei Street, Hsinchu 300, Taiwan \\ ${ }^{9}$ Department of Early Childhood Care and Education, Kang-Ning Junior College of Medical Care and Management, \\ No. 137, Lane 75, Section 3, Kangning Road, Neihu District, Taipei 114, Taiwan \\ ${ }^{10}$ Department of Nursing, School of Medicine, National Taiwan University, No. 1, Section 1, Jen Ai Road, \\ Zhongzheng District, Taipei 100, Taiwan \\ ${ }^{11}$ Department of Nursing, Ching-Kuo Institute of Management and Health, No. 336, Fu-Hsing Road, Zhongshan District, \\ Keelung 203, Taiwan \\ ${ }^{12}$ Department of Health Industry Management, School of Health Care Management, Kainan University, No. 1, \\ Kainan Road, Luzhu Shiang, Taoyuan 338, Taiwan
}

Correspondence should be addressed to Chia-Hsien Lin; g870615@gmail.com

Received 7 April 2014; Revised 12 May 2014; Accepted 12 May 2014; Published 3 June 2014

Academic Editor: Li-Yeh Chuang

Copyright (C) 2014 Shu-Chen Chien et al. This is an open access article distributed under the Creative Commons Attribution License, which permits unrestricted use, distribution, and reproduction in any medium, provided the original work is properly cited.

Jia-wei-xiao-yao-san (JWXYS) is a traditional Chinese herbal medicine that is widely used to treat neuropsychological disorders. Only a few of the hepatoprotective effects of JWXYS have been studied. The aim of this study was to investigate the hepatoprotective effects of JWXYS on dimethylnitrosamine- (DMN-) induced chronic hepatitis and hepatic fibrosis in rats and to clarify the mechanism through which JWXYS exerts these effects. After the rats were treated with DMN for 3 weeks, serum glutamic oxaloacetic transaminase (SGOT) and serum glutamic pyruvic transaminase (SGPT) levels were significantly elevated, whereas the albumin level decreased. Although DMN was continually administered, after the 3 doses of JWXYS were orally administered, the SGOT and SGPT levels significantly decreased and the albumin level was significantly elevated. In addition, JWXYS treatment prevented liver fibrosis induced by DMN. JWXYS exhibited superoxide-dismutase-like activity and dose-dependently inhibited DMN-induced lipid peroxidation and xanthine oxidase activity in the liver of rats. Our findings suggest that JWXYS exerts antifibrotic effects against DMN-induced chronic hepatic injury. The possible mechanism is at least partially attributable to the ability of JWXYS to inhibit reactive-oxygen-species-induced membrane lipid peroxidation. 


\section{Introduction}

The benefits of Chinese herbal medicines in treating chronic diseases, including chronic liver disease, have recently attracted the attention of Western practitioners. Jia-wei-xiaoyao-san (Kami-shoyo-san; TJ-24, JWXYS) is a traditional complex Chinese herbal medicine consisting of 10 medicinal herbal preparations. JWXYS is an officially approved prescription drug in China and Taiwan. Although some reports have addressed the neuropsychological activities of JWXYS, its hepatoprotective effects have not been adequately clarified $[1,2]$. The aim of this study was to investigate the hepatoprotective effects of JWXYS and to evaluate the mechanism through which JWXYS exerts these effects.

To investigate the hepatoprotective effect of JWXYS, chronic liver injury was induced by dimethylnitrosamine (DMN) [3-6]. Studies have reported that DMN can induce lipid peroxidation in the liver, thereby reducing hepatic tissue blood flow and leading to acute liver damage and even fulminant hepatitis $[7,8]$.

In the present study, we examined the hepatoprotective effect of JWXYS by determining the levels of serum glutamic oxaloacetic transaminase (SGOT), serum glutamic pyruvic transaminase (SGPT), albumin in the serum, and histopathological changes in rat hepatic tissues. The antioxidative effects of JWXYS on liver tissues and the superoxide-dismutase(SOD-) like activity of JWXYS were evaluated to determine the possible mechanism.

\section{Methods}

2.1. Drugs and Chemicals. JWXYS (Kami-shoyo-san; TJ-24) was provided by Koda Pharmaceutical (Taoyuan, Taiwan). It consists of 10 medicinal herb preparations, namely, Angelica sinensis radix (3.0 g), Bupleurum falcatum radix (3.0 g), Paeonia albiflora (3.0 g), glycyrrhizae radix (2.0 g), Moutan Radicis cortex (2.0 g), Gardenia fructus (2.0 g), Zingiber officinale rhizome (1.0 g), Atractylodis macrocephalae rhizome $(3.0 \mathrm{~g})$, Poria cocos (3.0 g), and Mentha arvensis (1.0 g). JWXYS was dissolved in $0.9 \% \mathrm{NaCl}$ at concentrations of 100,300 , and $1000 \mathrm{mg} / 2 \mathrm{~mL}$ before use.

DMN and silymarin were purchased from Sigma (St. Louis, MO, USA). Before use, DMN was dissolved in $0.9 \%$ $\mathrm{NaCl}$ at a concentration of $1 \%$, and silymarin was dissolved in $1 \%$ carboxymethylcellulose at a concentration of $200 \mathrm{mg} / 2 \mathrm{~mL}$.

2.2. Animals. Male Wistar rats (110-130 g; BioLasco Taiwan, Taiwan) were used. The Institutional Review Board at China Medical University (Taichung, Taiwan) reviewed and approved the study protocols. The animals were allowed to acclimate for at least 7 days on a standard laboratory diet (Fu-So, Taipei, Taiwan) under environmentally controlled conditions $\left(25 \pm 1^{\circ} \mathrm{C}\right.$ and $55 \% \pm 5 \%$ humidity) with free access to food and tap water. A $12 \mathrm{~h}$ light/dark cycle was maintained, and hardwood chips were used as bedding.

In this study, 60 rats weighing $160-240 \mathrm{~g}$ were randomly divided into 6 groups: control (normal saline-treated),
DMN-treated, DMN-treated + silymarin $(200 \mathrm{mg} / \mathrm{kg})$, and DMN-treated + JWXYS $(100,300$, and $1000 \mathrm{mg} / \mathrm{kg})$ groups.

2.3. Dimethylnitrosamine-Induced Liver Injury and Treatments. Dimethylnitrosamine (DMN) can induce hepatic sinusoidal endothelial injury and coagulation necrosis primarily in the central and periportal regions of the lobule $[9,10]$. It is usually used to induce experimental liver fibrosis [4]. In our study, DMN was dissolved in normal saline and then intraperitoneally (i.p.) administered to rats 3 times per week at doses of $10 \mathrm{mg} / \mathrm{mL} / \mathrm{kg}$. After 3 weeks of inducing liver damage, DMN was continually i.p. administered to the 5 experimental groups, but not to the normal saline control group $(1 \mathrm{~mL} / \mathrm{kg})$, for the following 3 weeks. From the beginning of the subsequent 3 -week period, JWXYS $(100,300$, and $1000 \mathrm{mg} / 2 \mathrm{~mL} / \mathrm{kg})$ was orally administered 3 times per day on 3 days in each week to 3 experimental groups. Before the rats were sacrificed, they were starved for $24 \mathrm{~h}$ after the final oral administration of JWXYS. Silymarin $(200 \mathrm{mg} / 2 \mathrm{~mL} / \mathrm{kg}$ ) was orally administered to the silymarin group 3 times per day for 3 weeks.

2.4. Serum Biochemical and Pathological Evaluation. The protective effect of JWXYS against DMN-induced liver injury was evaluated by assessing the SGOT, SGPT, and serum albumin levels and by examining histopathological sections of the liver of all experimental animals [7].

2.5. Antioxidative Effect of Jia-Wei-Xiao-Yao-San. A study reported that $(+)$-alpha-tocopherol (vitamin E) can scavenge DMN-induced superoxide-free radical production [11]. Therefore, vitamin $\mathrm{E}$ was used as a positive control in this study.

To compare the antioxidative effects of JWXYS and vitamin $\mathrm{E}(0.5 \mathrm{mM})$ (as a positive control) in rat liver homogenates, $\mathrm{FeCl}_{2}$-induced lipid peroxidation was determined based on the formation of a malonic dialdehyde(MDA-) thiobarbituric acid (TBA) product according to a modified method described by Yuda et al. [12].

Furthermore, to evaluate the inhibitory activity of JWXYS against DMN-induced lipid peroxidation, MDA-TBA products of JWXYS- $(100,300$, and $1000 \mathrm{mg} / \mathrm{kg})$ treated rat livers were measured using the method described by Yuda et al. [12].

2.6. Superoxide-Dismutase-Like Activity Test. To evaluate the superoxide-scavenging activity of JWXYS, a cytochrome C reduction method developed by $\mathrm{McC}$ ord and Fridovich was used [13]. Each data point represents the percent of superoxide inhibition (SI, \%), and each assay was conducted in triplicate. Xanthine oxidase (XOD) converts xanthine to uric acid, yielding superoxide anions as a byproduct and subsequently converting ferricytochrome $\mathrm{C}$ directly to ferrocytochrome $\mathrm{C}$, which exhibits absorbance at $550 \mathrm{~nm}$. When a compound exhibits superoxide-scavenging activity, a reduction in ferricytochrome $\mathrm{C}$ occurs. Inhibition of XOD can reduce the production of superoxide anions, and XOD inhibition was measured according to the method described by Frederiks and Bosch [14]. 
TABLE 1: Effects of JWXYS and silymarin on the body weights of rats with DMN-induced chronic liver injury.

\begin{tabular}{|c|c|c|c|c|c|}
\hline \multicolumn{2}{|r|}{ Group } & \multicolumn{4}{|c|}{ Time after DMN treatment (day) } \\
\hline & & 0 & 14 & 28 & 42 \\
\hline $\bar{A}$ & Normal control & $148.0 \pm 12.2$ & $214.0 \pm 13.3$ & $282.0 \pm 33.9$ & $348.8 \pm 35.5$ \\
\hline B & DMN (10 mg/kg) & $143.7 \pm 23.3$ & $190.7 \pm 19.3$ & $225.7 \pm 10.5$ & $265.7 \pm 11.0^{*}$ \\
\hline $\mathrm{C}$ & DMN $(10 \mathrm{mg} / \mathrm{kg})+$ silymarin $(200 \mathrm{mg} / \mathrm{kg})$ & $145.0 \pm 20.0$ & $193.2 \pm 16.8$ & $273.2 \pm 22.0$ & $325.2 \pm 28.7$ \\
\hline $\mathrm{D}$ & DMN $(10 \mathrm{mg} / \mathrm{kg})+\mathrm{JWXYS}(100 \mathrm{mg} / \mathrm{kg})$ & $144.7 \pm 17.3$ & $190.7 \pm 16.9$ & $272.7 \pm 16.3$ & $324.8 \pm 16.2$ \\
\hline $\mathrm{E}$ & DMN (10 mg/kg) + JWXYS (300 mg/kg) & $145.0 \pm 16.9$ & $190.2 \pm 16.9$ & $273.0 \pm 16.4$ & $325.6 \pm 17.2$ \\
\hline $\mathrm{F}$ & DMN (10 mg/kg) + JWXYS (1000 mg/kg) & $145.0 \pm 20.0$ & $195.2 \pm 16.9$ & $277.0 \pm 17.2$ & $331.8 \pm 18.2^{\#}$ \\
\hline
\end{tabular}

Each value is presented as the mean $\pm \operatorname{SE}(n=10)$.

${ }^{*} P<0.05$, significantly different from Group A.

${ }^{\#} P<0.05$, significantly different from Group B.

2.7. Statistical Analysis. Statistical significance was calculated by conducting a one-way analysis of variance coupled with Dunnett's test. $P$ values less than 0.05 indicated statistical significance.

\section{Results}

3.1. Body Weight. As shown in Table 1, after treatment with DMN for 42 days, the body weights of rats significantly decreased $(P<0.05)$. By contrast, the body weights of the rats significantly increased $(P<0.05)$ after they were treated with JWXYS $(1000 \mathrm{mg} / \mathrm{kg})$ in the second 3-week period compared with those of the group treated with only DMN.

3.2. Histological Observations. Histological observations confirmed the hepatoprotective effect of JWXYS. Figure 1(a) shows a normal control rat liver, in which no necrosis or inflammation was observed. By contrast, the liver of rats treated with $10 \mathrm{mg} / \mathrm{kg}$ of DMN exhibited marked widening, inflammation, and a fibrotic portal area, with irregular borders forming a spiked appearance and portal-bridging fibrosis. Nodular transformation was apparent (Figure 1(b)). In the liver of silymarin- $(200 \mathrm{mg} / \mathrm{kg})$ treated rats, only moderate widening and a fibrotic portal area with bridging fibrosis were observed (Figure 1(c)).

The protective effect of JWXYS (100, 300, and $1000 \mathrm{mg} / \mathrm{kg}$ ) against DMN-induced chronic liver injury was determined by examining histological changes in rat livers. The livers of the rats in the JWXYS $(100 \mathrm{mg} / \mathrm{kg})$ group exhibited moderate widening and a fibrotic portal area with bridging and mild inflammatory cell infiltration, which were also observed in the silymarin- $(200 \mathrm{mg} / \mathrm{kg})$ treated group (Figure 1(d)). By contrast, in the livers of rats treated with 300 and $1000 \mathrm{mg} / \mathrm{kg}$ of JWXYS, only mild focal fibrotic changes of the portal area and mild bridging were noted (Figures $1(\mathrm{e})$ and $1(\mathrm{f}))$. Thus, histological improvements in the livers of rats treated with 300 and $1000 \mathrm{mg} / \mathrm{kg}$ of JWXYS were obviously more substantial than those in the livers of rats treated with $100 \mathrm{mg} / \mathrm{kg}$ of JWXYS or $200 \mathrm{mg} / \mathrm{kg}$ of silymarin.

3.3. Serum Biochemical Assay. As shown in Table 2, the SGOT and SGPT levels of the experimental groups were significantly elevated, whereas the albumin level decreased after DMN treatment compared with those of the normal control group. The results indicated that DMN can induce chronic active hepatitis and diffuse liver injury.

By contrast, treatment with JWXYS (100, 300, and $1000 \mathrm{mg} / 2 \mathrm{~mL} / \mathrm{kg})$ or silymarin $(200 \mathrm{mg} / 2 \mathrm{~mL} / \mathrm{kg})$ during the second 3-week period significantly prevented further DMNinduced elevations in serum GOT and GPT levels and a further decrease in the serum albumin level (Table 2). According to these results, JWXYS exhibited strong hepatoprotective effects against DMN-induced chronic hepatic injury in rats. The hepatoprotective ability of 300 and $1000 \mathrm{mg} / \mathrm{kg}$ of JWXYS was greater than that of silymarin alone.

3.4. $\mathrm{FeCl}_{2}$-Stimulated Lipid Peroxidation In Vitro. Although JWXYS $(10 \mathrm{mg} / \mathrm{kg})$ significantly inhibited $51.71 \%$ of $\mathrm{FeCl}_{2}$ stimulated lipid peroxidation in vitro, $0.5 \mathrm{mM}$ vitamin $\mathrm{E}$ inhibited $71.10 \%$ of $\mathrm{FeCl}_{2}$-stimulated lipid peroxidation. JWXYS $(0.1,1.0$, and $10 \mathrm{mg} / \mathrm{kg})$ significantly and dosedependently inhibited $\mathrm{FeCl}_{2}$-stimulated lipid peroxidation in vitro. Nevertheless, we proved that JWXYS (100, 300, and $1000 \mathrm{mg} / \mathrm{kg}$ ) dose-dependently inhibited DMN-induced lipid peroxidation in rat livers (in vivo). No significant difference was observed between treatments with $1000 \mathrm{mg} / \mathrm{kg}$ of JWXYS (69.00\%) and $0.69 \mathrm{mM}$ vitamin $\mathrm{E}(71.16 \%)$ (Table 3$)$.

3.5. Superoxide-Dismutase-Like Activity. The SOD-like activity (SI\%) of JWXYS (0.01, 0.1, and $1.0 \mathrm{mg} / \mathrm{mL}$ ) was examined to clarify the superoxide-scavenging ability. The activity of vitamin E $(8 \mathrm{mg} / \mathrm{kg})$ was set to $100 \%$ of SOD-like activity. The results indicated that the SOD-like activity levels of JWXYS at $0.01,0.1$, and $1.0 \mathrm{mg} / \mathrm{mL}$ were $33.77 \%$, $68.83 \%$, and $94.81 \%$, respectively. JWXYS at $1.0 \mathrm{mg} / \mathrm{mL}$ exhibited the strongest superoxide-scavenging activity (94.81\%) (Table 4).

XOD converts xanthine to uric acid, thus yielding superoxide anions as a byproduct [15]. Therefore, inhibition of XOD can reduce the production of superoxide anions. As shown in Table 4, the levels of XOD inhibition (SI\%) induced by JWXYS at $0.01,0.1$, and $1.0 \mathrm{mg} / \mathrm{mL}$ were $11.90 \%, 14.29 \%$, and $83.33 \%$, respectively. These results showed that JWXYS dose-dependently inhibited XOD activity. 


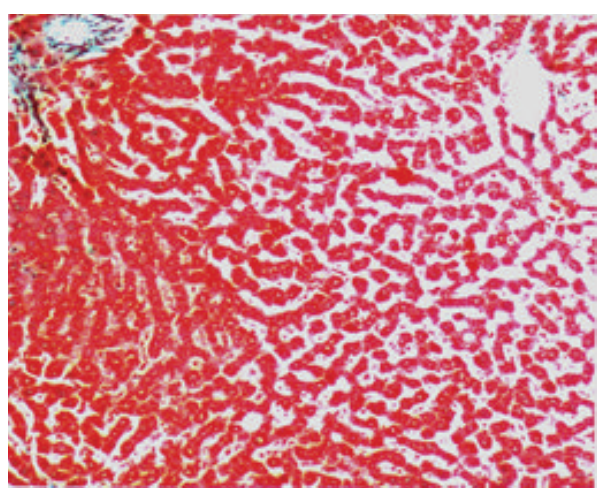

(a)

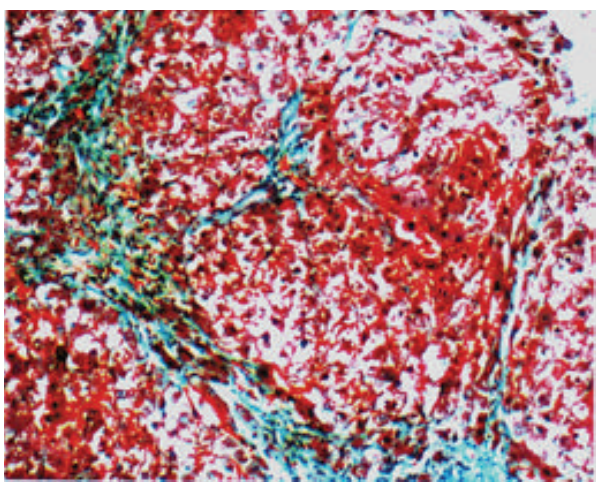

(c)

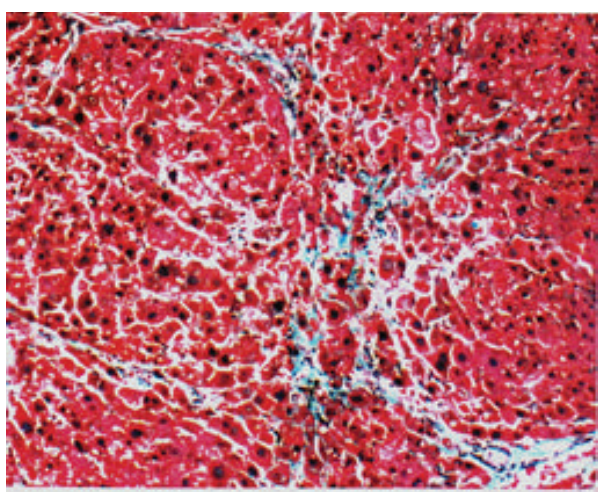

(e)

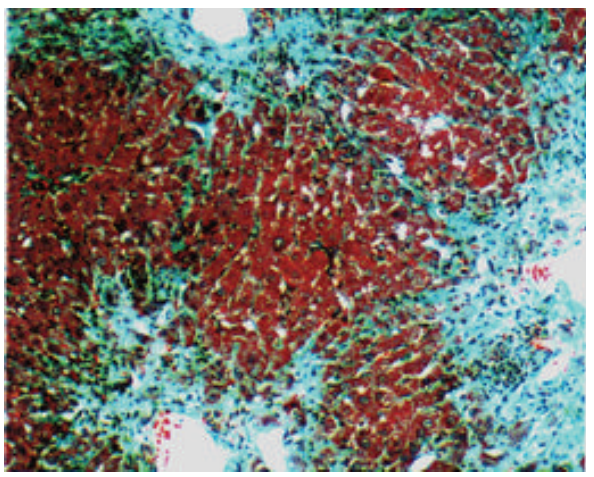

(b)

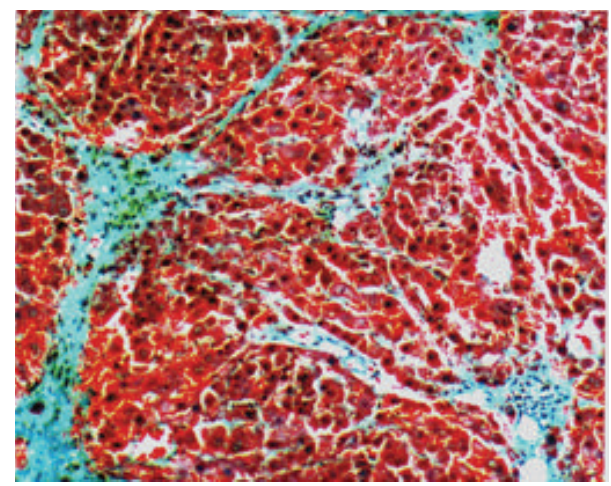

(d)

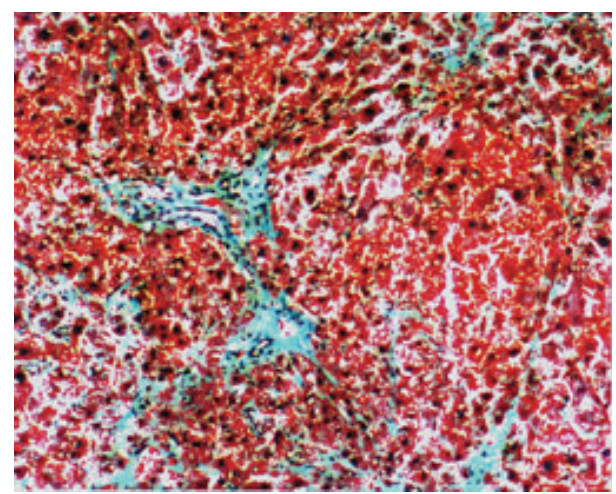

(f)

FIGURE 1: Histological examination performed by conducting Masson trichrome staining, 100x of JWXYS-treated and untreated rat liver. (a) vehicle, normal saline; control, normal liver; (b) DMN alone with marked widening, inflammation, and fibrotic portal tracts with bridging fibrosis. Nodular transformation was apparent. (c) DMN $+200 \mathrm{mg} / \mathrm{kg}$ of silymarin with moderate widening and fibrotic portal tracts with occasional bridging fibrosis; (d) DMN $+100 \mathrm{mg} / \mathrm{kg}$ of JWXYS with moderate widening and fibrotic portal tracts with bridging fibrosis and mild inflammatory cell infiltration; (e) DMN + $300 \mathrm{mg} / \mathrm{kg}$ of JWXYS with focal mild fibrotic change of the portal tracts and bridging fibrosis; (f) $\mathrm{DMN}+1000 \mathrm{mg} / \mathrm{kg}$ of JWXYS with mild to moderate fibrotic portal tracts with bridging fibrosis.

\section{Discussion}

Chronic hepatitis $\mathrm{B}$, hepatitis $\mathrm{C}$, and alcoholism are the major risk factors for developing liver cirrhosis and hepatocellular carcinoma. The pathway for the progression to liver cirrhosis is the fibrotic process in the liver of patients with the aforementioned risk factors $[16,17]$. Therefore, antifibrotic therapy for chronic hepatitis is a crucial topic. A previous study revealed that the combination of interferon and ribavirin exerted an antifibrotic effect in treating chronic hepatitis $\mathrm{C}$. However, the effect was limited, and concerns have been raised regarding the costs and side effects [18-20]. Studies have suggested that alternative agents such as silymarin, shosaiko-to, halofuginone, imatinib mesylate, phosphodiesterase inhibitors, and endothelin-A-receptor and angiotensin antagonists have antifibrotic effects in chronic hepatitis [20,21].

Shimizu et al. reported that the traditional Chinese herbal medicine sho-saiko-to (as it is called in Japanese), which 
TABLE 2: Effects of JWXYS on the SGOT, SGPT, and albumin levels in rats with DMN-induced chronic liver injury.

\begin{tabular}{|c|c|c|c|c|}
\hline & Group & $\begin{array}{l}\text { SGOT } \\
(\mathrm{IU} / \mathrm{L})\end{array}$ & $\begin{array}{l}\text { SGPT } \\
(\mathrm{IU} / \mathrm{L})\end{array}$ & $\begin{array}{c}\text { Albumin } \\
(\mathrm{g} / \mathrm{dL})\end{array}$ \\
\hline A & Normal control & $105.3 \pm 13.9$ & $24.2 \pm 3.3$ & $4.45 \pm 0.02$ \\
\hline B & DMN (10 mg/kg) & $320.1 \pm 42.3^{\# \#}$ & $80.3 \pm 11.8^{\# \#}$ & $3.24 \pm 0.04^{\#}$ \\
\hline $\mathrm{C}$ & DMN $(10 \mathrm{mg} / \mathrm{kg})+$ silymarin $(200 \mathrm{mg} / \mathrm{kg})$ & $200.4 \pm 17.6^{* *}$ & $66.7 \pm 11.1^{* *}$ & $3.87 \pm 0.14^{* *}$ \\
\hline $\mathrm{D}$ & DMN (10 mg/kg) + JWXYS (100 mg/kg) & $211.3 \pm 15.6^{* *}$ & $70.1 \pm 7.6^{*}$ & $3.76 \pm 0.15$ \\
\hline $\mathrm{E}$ & DMN (10 mg/kg) + JWXYS (300 mg/kg) & $193.6 \pm 11.8^{* *}$ & $63.3 \pm 7.3^{* *}$ & $3.86 \pm 0.16^{* *}$ \\
\hline $\mathrm{F}$ & DMN (10 mg/kg) + JWXYS (1000 mg/kg) & $168.3 \pm 9.9^{* *}$ & $47.3 \pm 6.8^{* *}$ & $3.96 \pm 0.18^{* *}$ \\
\hline
\end{tabular}

Values are presented as the mean $\pm \mathrm{SE}(n=10)$.

${ }^{\#, \# \# P} P<0.05$ and 0.01 , significantly different from Group A.

${ }^{*, * *} P<0.05$ and 0.01 , significantly different from Group B.

One-way analysis of variance coupled with Dunnett's test.

$P$ values $<0.05$ indicated significance.

TABLE 3: Inhibitory effects of various doses of JWXYS on $\mathrm{FeCl}_{2}$ induced (in vitro) and $\mathrm{DMN}$-induced (in vivo) lipid peroxidation in rat livers.

\begin{tabular}{|c|c|c|c|}
\hline \multicolumn{2}{|r|}{ Group } & \multirow{2}{*}{$\begin{array}{c}\text { MDA } \\
\left(\begin{array}{c}\text { nmole/mg } \\
\text { protein) }\end{array}\right. \\
1.69 \pm 0.11\end{array}$} & \multirow[t]{2}{*}{$\begin{array}{c}\text { Inhibition } \\
\text { rate }(\%)\end{array}$} \\
\hline $\mathrm{A}$ & Normal control & & \\
\hline \multicolumn{4}{|c|}{ In vitro } \\
\hline B & $\mathrm{FeCl}_{2}$ & $2.63 \pm 0.08^{* *}$ & \\
\hline $\mathrm{C}$ & $\mathrm{FeCl}_{2}+\operatorname{vitamin} \mathrm{E}(0.5 \mathrm{mM})$ & $0.76 \pm 0.05^{\# \#}$ & 71.10 \\
\hline $\mathrm{D}$ & $\mathrm{FeCl}_{2}+\mathrm{JWXYS}(0.1 \mathrm{mg} / \mathrm{kg})$ & $2.10 \pm 0.05^{\# \#}$ & 20.15 \\
\hline $\mathrm{E}$ & $\mathrm{FeCl}_{2}+\mathrm{JWXYS}(1.0$ mg/kg) & $1.44 \pm 0.06^{\# \#}$ & 45.25 \\
\hline $\mathrm{F}$ & $\mathrm{FeCl}_{2}+\mathrm{JWXYS}(10 \mathrm{mg} / \mathrm{kg})$ & $1.27 \pm 0.03^{\# \# \#}$ & 51.71 \\
\hline \multicolumn{4}{|c|}{ In vivo } \\
\hline G & $\mathrm{DMN}$ & $3.71 \pm 0.05^{* *}$ & \\
\hline $\mathrm{H}$ & $\mathrm{DMN}+\operatorname{vitamin} \mathrm{E}(0.69 \mathrm{mM})$ & $1.07 \pm 0.05^{\triangle \Delta \Delta}$ & 71.16 \\
\hline I & DMN + JWXYS (100 mg/kg) & $2.79 \pm 0.05^{\triangle \Delta}$ & 24.80 \\
\hline $\mathrm{J}$ & DMN + JWXYS (300 mg/kg) & $2.21 \pm 0.06^{\triangle \triangle}$ & 40.43 \\
\hline $\mathrm{K}$ & DMN + JWXYS (1000 mg/kg) & $1.15 \pm 0.03^{\triangle \Delta \Delta}$ & 69.00 \\
\hline
\end{tabular}

Each value is presented as the mean $\pm \mathrm{SE}(n=6)$. Vitamin $\mathrm{E}$ was used as the positive control.

${ }^{* *} P<0.01$, significantly different from Group A.

$\# \#, \# \#+P<0.01$ and 0.001 , significantly different from Group B.

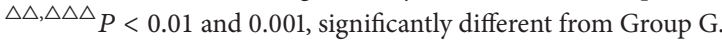

One-way analysis of variance coupled with Dunnett's test.

$P$ values $<0.05$ indicated significance.

features effects similar to those of silibinin, exhibited radicalscavenging ability and antifibrotic properties in activated hepatic stellate cells in vitro and in porcine serum-induced fibrosis in vivo [22]. Like sho-saiko-to, JWXYS is an option for treating patients with chronic hepatitis in China and other Asian countries.

As shown in Table 1, JWXYS eliminated the effect of DMN-induced body weight loss in rats. In addition, we observed that JWXYS treatment inhibited DMN-induced hepatic injury and fibrosis in a dose-dependent manner. The effects of JWXYS were stronger than that of silymarin (Figures 1(e) and 1(f)). As shown in Table 2, JWXYS significantly diminished the DMN-induced hepatitis and severe diffuse
TABLE 4: Effects of JWXYS on SOD-like activity and XOD inhibition.

\begin{tabular}{cccc}
\hline Group & $\begin{array}{c}\text { SOD-like } \\
\text { activity } \\
(\text { SI\% })^{*}\end{array}$ & $\begin{array}{c}\text { XOD } \\
\text { inhibition } \\
(\text { SI\% })^{\#}\end{array}$ \\
\hline A & Vitamin E $(8 \mathrm{mg} / \mathrm{kg})$ & 100.00 & \\
B & JWXYS $(0.01 \mathrm{mg} / \mathrm{mL})$ & 33.77 & 11.90 \\
C & JWXYS $(0.1 \mathrm{mg} / \mathrm{mL})$ & 68.83 & 14.29 \\
D & JWXYS $(1.0 \mathrm{mg} / \mathrm{mL})$ & 94.81 & 83.33 \\
\hline
\end{tabular}

${ }^{*}$ Measured using the cytochrome $\mathrm{C}$ reduction test.

${ }^{\#}$ Measured using the XOD inhibition test.

Vitamin $\mathrm{E}$ was used as the positive control.

SI: superoxide inhibition.

liver injury, thereby disrupting the sequence of events leading to liver fibrosis.

Recent research has indicated that chronic liver injury and hepatic fibrosis are related to oxidative stress, including that caused by reactive oxygen species (ROS) and lipid peroxidation $[23,24]$. Studies have suggested that antioxidants such as SOD, catalase, and estradiol significantly prevent lipid peroxidation and exacerbation of liver fibrosis [25-27]. Indeed, as shown in Table 4, JWXYS inhibited XOD activity in a dose-dependent manner. XOD converts xanthine to uric acid, yielding superoxide anions as a byproduct [15], and the inhibition of XOD can substantially reduce the production of superoxide anions, thus reducing superoxide-induced lipid peroxidation and subsequent liver fibrosis.

Furthermore, our study revealed that the Chinese herbal medicine, JWXYS, significantly inhibited $\mathrm{FeCl}_{2}$-stimulated (in vitro) and $\mathrm{DMN}$-induced (in vitro) lipid peroxidation in a dose-dependent manner (Table 3 ). In the cytochrome $C$ test, we confirmed that JWXYS exhibits a dose-dependent antioxidative activity (Table 4). Furthermore, in another experiment we performed, JWXYS exhibited no hepatotoxic effect in Wistar rats even at a dose of $2000 \mathrm{mg} / \mathrm{kg}$ orally administered for 6 weeks (data not shown).

Our study has limitations because, to extrapolate conclusions regarding humans from experimental data on rats, the dose must be validated in clinical trials. The mechanism through which JWXYS acts in cellular pathways remains 
undetermined. Immunohistochemical analysis of markers such as alpha-smooth muscle actin in activated hepatic stellate cells and sets of in vitro culture studies on parenchymal cells and nonparenchymal cells might provide additional evidence supporting the molecular mechanisms through which JWXYS exerts antifibrotic effects. Moreover, in an ongoing follow-up study conducted in our laboratory, JWXYS exhibited strong free-radical-scavenging and antioxidant activity and inhibited free-radical-induced hepatic fibrosis. We will continue to conduct research on this topic.

\section{Conclusions}

We reported that JWXYS exhibits obvious dose-dependent antifibrotic effects against chronic hepatic injury that are similar to those exhibited by sho-saiko-to. This protective effect was noted even in a condition in which DMN was continually administered. The mechanism may at least partially be due to the inhibitory effect of JWXYS on lipid peroxidation and its superoxide-scavenging activities. We hope that, after additional evidence has been accumulated, JWXYS will be applied as an alternative clinical medication for chronic hepatitis and hepatic antifibrotic therapy because of its efficiency, low cost, and fewer side effects.

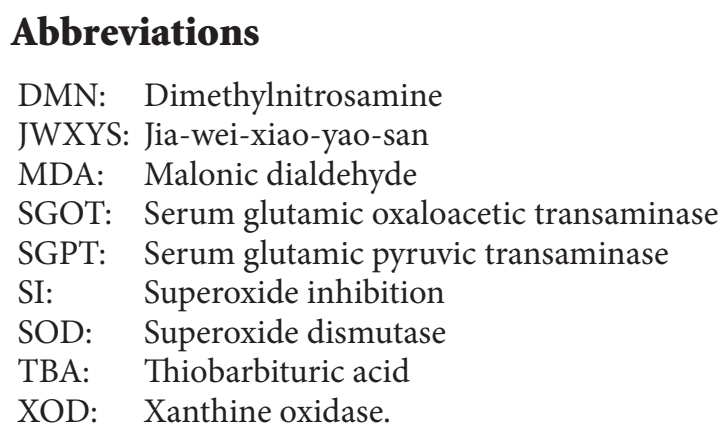

\section{Conflict of Interests}

The authors declare that there is no conflict of interests regarding the publication of this paper.

\section{Authors' Contribution}

Shu-Chen Chien and Wei-Chiao Chang contributed to this work equally.

\section{Acknowledgment}

This work was supported by grants (NSC101-2320-B-424001) provided by the National Science Council, Taiwan, and Health and welfare surcharge of tobacco products, Taiwan.

\section{References}

[1] T. Ishikawa, T. Funahashi, and J. Kudo, "Effectiveness of the Kampo kami-shoyo-san (TJ-24) for tremor of antipsychoticinduced parkinsonism," Psychiatry \& Clinical Neurosciences, vol. 54, no. 5, pp. 579-582, 2000.
[2] K. Yamada and S. Kanba, "Herbal medicine (Kami-shoyo-san) in the treatment of premenstrual dysphoric disorder," Journal of Clinical Psychopharmacology, vol. 22, no. 4, p. 442, 2002.

[3] J.-Y. Chen, H.-L. Chen, J.-C. Cheng et al., "A Chinese herbal medicine, Gexia-Zhuyu Tang (GZT), prevents dimethylnitrosamine-induced liver fibrosis through inhibition of hepatic stellate cells proliferation," Journal of Ethnopharmacology, vol. 142, no. 3, pp. 811-818, 2012.

[4] J. Fujimoto, "Gene therapy for liver cirrhosis," Journal of Gastroenterology \& Hepatology, vol. 15, pp. D33-D36, 2000.

[5] M. Kusunose, B. Qiu, T. Cui et al., "Effect of Sho-saiko-to extract on hepatic inflammation and fibrosis in dimethylnitrosamine induced liver injury rats," Biological and Pharmaceutical Bulletin, vol. 25, no. 11, pp. 1417-1421, 2002.

[6] Y. Matsuda, K. Matsumoto, T. Ichida, and T. Nakamura, "Hepatocyte growth factor suppresses the onset of liver cirrhosis and abrogates lethal hepatic dysfunction in rats," Journal of Biochemistry, vol. 118, no. 3, pp. 643-649, 1995.

[7] S. C. Lin, C. C. Lin, Y. H. Lin, and C. H. Chen, "Protective and therapeutic effects of ban-zhi-lian on hepatotoxin-induced liver injuries," American Journal of Chinese Medicine, vol. 22, no. 1, pp. 29-42, 1994.

[8] A. Suzuki, M. Hagino, N. Yasuda et al., "Inhibitory effects of prostaglandin E1- $\alpha$-cyclodextrin (PGE1·CD) on dimethylnitrosamine-induced acute liver damage in rats," Folia Pharmacologica Japonica, vol. 105, no. 4, pp. 221-229, 1995.

[9] A. M. Jézéquel, R. Mancini, M. L. Rinaldesi, G. Macarri, C. Venturini, and F. Orlandi, "A morphological study of the early stages of hepatic fibrosis induced by low doses of dimethylnitrosamine in the rat," Journal of Hepatology, vol. 5, no. 2, pp. 174-181, 1987.

[10] X. Lu, P. Liu, C. Liu et al., "Role of hepatic sinusoidal endothelium injury in hepatic fibrogenesis induced by dimethylnitrosamine in rats," Zhonghua Gan Zang Bing Za Zhi, vol. 10, no. 6, pp. 441-444, 2002.

[11] D. Wu and A. I. Cederbaum, "Ethanol cytotoxicity to a transfected HepG2 cell line expressing human cytochrome P4502E1," Journal of Biological Chemistry, vol. 271, no. 39, pp. 23914-23919, 1996.

[12] Y. Yuda, J. Tanaka, F. Hirano, K. Igarashi, and T. Satoh, "Participation of lipid peroxidation in rat pertussis vaccine pleurisy. III. Thiobarbituric acid (TBA) reactant and lysosomal enzyme," Chemical and Pharmaceutical Bulletin, vol. 39, no. 2, pp. 505-506, 1991.

[13] J. M. McCord and I. Fridovich, "The reduction of cytochrome c by milk xanthine oxidase," Journal of Biological Chemistry, vol. 243, no. 21, pp. 5753-5760, 1968.

[14] W. M. Frederiks and K. S. Bosch, "The role of xanthine oxidase in ischemia/reperfusion damage of rat liver," Histology \& Histopathology, vol. 10, no. 1, pp. 111-116, 1995.

[15] E. C. Viel, K. Benkirane, D. Javeshghani, R. M. Touyz, and E. L. Schiffrin, "Xanthine oxidase and mitochondria contribute to vascular superoxide anion generation in DOCA-salt hypertensive rats," American Journal of Physiology: Heart and Circulatory Physiology, vol. 295, no. 1, pp. H281-H288, 2008.

[16] T. Poynard, P. Bedossa, and P. Opolon, "Natural history of liver fibrosis progression in patients with chronic hepatitis C," The Lancet, vol. 349, no. 9055, pp. 825-832, 1997.

[17] Y. Wang, M.-Y. Xu, R.-D. Zheng et al., "Prediction of significant fibrosis and cirrhosis in hepatitis B e-antigen negative patients with chronic hepatitis B using routine parameters," Hepatology Research, vol. 43, no. 5, pp. 441-451, 2013. 
[18] G. S. Baroni, L. D’Ambrosio, P. Curto et al., "Interferon gamma decreases hepatic stellate cell activation and extracellular matrix deposition in rat liver fibrosis," Hepatology, vol. 23, no. 5, pp. 1189-1199, 1996.

[19] G. Giannelli, C. Bergamini, F. Marinosci et al., "Antifibrogenic effect of IFN- $\alpha 2$ b on hepatic stellate cell activation by human hepatocytes," Journal of Interferon \& Cytokine Research, vol. 26, no. 5, pp. 301-308, 2006.

[20] D. Schuppan, A. Krebs, M. Bauer, and E. G. Hahn, "Hepatitis C and liver fibrosis," Cell Death \& Differentiation, vol. 10, no. 1, pp. S59-S67, 2003.

[21] H. Yoshiji, R. Noguchi, S. Kuriyama et al., "Imatinib mesylate (STI-571) attenuates liver fibrosis development in rats," American Journal of Physiology: Gastrointestinal \& Liver Physiology, vol. 288, no. 5, pp. G907-G913, 2005.

[22] I. Shimizu, Y.-R. Ma, Y. Mizobuchi et al., "Effects of Sho-saikoto, a Japanese herbal medicine, on hepatic fibrosis in rats," Hepatology, vol. 29, no. 1, pp. 149-160, 1999.

[23] P. Bedossa, K. Houglum, C. Trautwein, A. Holstege, and M. Chojkier, "Stimulation of collagen $\alpha 1$ (I) gene expression is associated with lipid peroxidation in hepatocellular injury: a link to tissue fibrosis?" Hepatology, vol. 19, no. 5, pp. 1262-1271, 1994.

[24] G. Vendemiale, I. Grattagliano, M. L. Caruso et al., "Increased oxidative stress in dimethylnitrosamine-induced liver fibrosis in the rat: effect of $\mathrm{N}$-acetylcysteine and interferon- $\alpha$," Toxicology and Applied Pharmacology, vol. 175, no. 2, pp. 130-139, 2001.

[25] G. Lu, I. Shimizu, X. Cui et al., "Antioxidant and antiapoptotic activities of idoxifene and estradiol in hepatic fibrosis in rats," Life Sciences, vol. 74, no. 7, pp. 897-907, 2004.

[26] K. S. Lee, M. Buck, K. Houglum, and M. Chojkier, "Activation of hepatic stellate cells by TGF $\alpha$ and collagen type I is mediated by oxidative stress through c-myb expression," The Journal of Clinical Investigation, vol. 96, no. 5, pp. 2461-2468, 1995.

[27] R. H. Shi, T. X. Chen, and X. Z. Lu, "Protective effects of free radical scavengers superoxide dismutase and catalase on experimental liver fibrosis," Zhonghua Xiaohua Zazhi, vol. 13, pp. 84-86, 1993. 

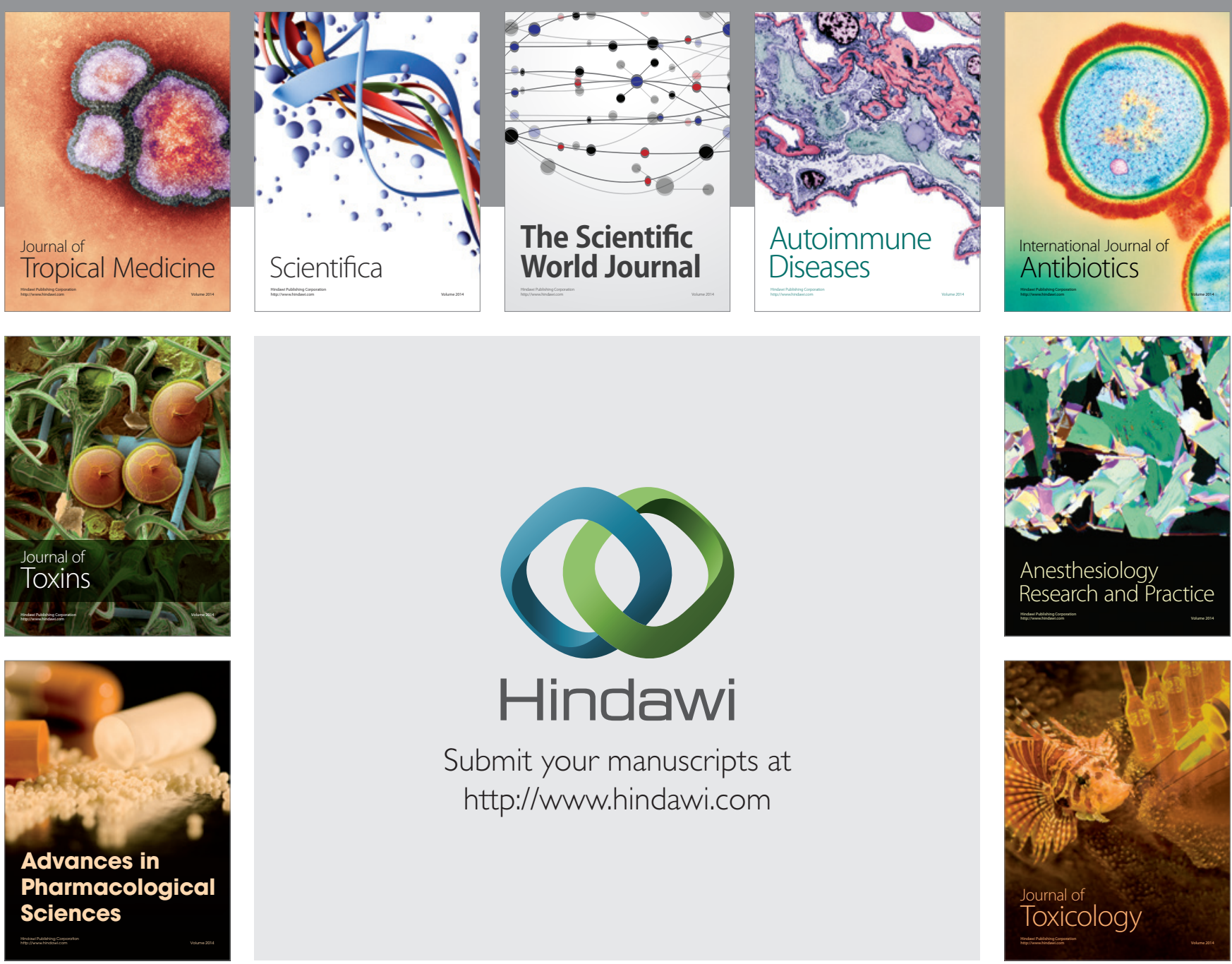

\section{Hindawi}

Submit your manuscripts at

http://www.hindawi.com
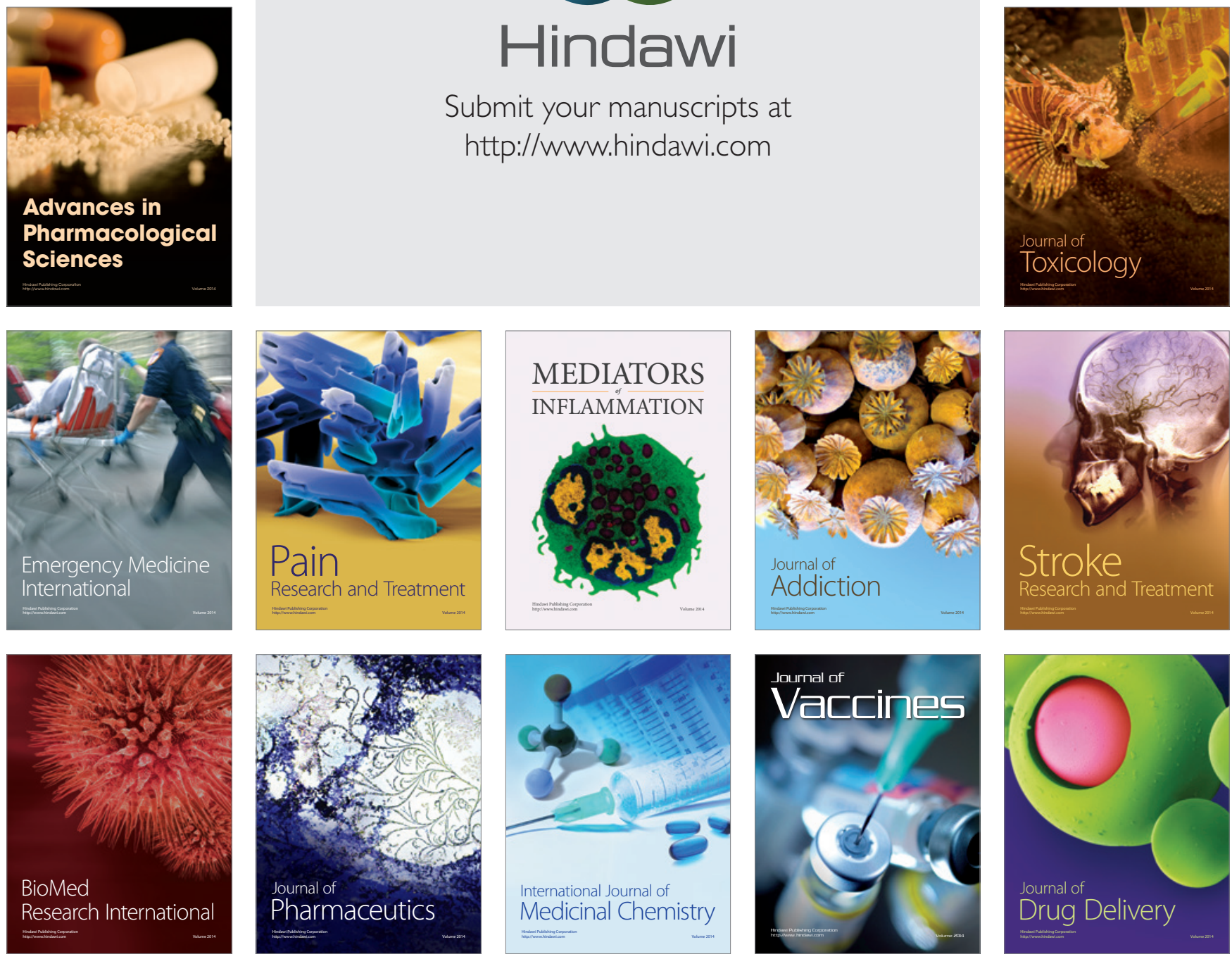